\title{
Athericidae d'Afrique du Nord. II. Ibisia amicorum n. sp. (Diptera, Brachycera).
}

\author{
A.G.B. Thomas 1
}

Mots clés : Diptera, Brachycera, Athericidae, imago or, nouvelle espèce, Maroc.

L'imago or d'Ibisia amicorum n. sp., proche d'I. marginata (Fabricius) et d'I. vaillanti Thomas est décrite et figurée. Cette espèce a été rencontrée dans le Sud du Maroc.

\section{Athericidae from North Africa :}

II. Ibisia amicorum n. sp. (Diptera, Brachycera).

Keywords : Diptera, Brachycera, Athericidae, imago o, new species, Marocco.

The male adult of Ibisia amicorum $\mathrm{n}$. sp., similar to those of $I$. marginata (Fabricius) and of $I$. vaillanti Thomas is described and illustrated. This species has been found in the South of Morocco.

Lors de la prospection de cours d'eau de haute altitude situés dans le massif montagneux du Jebel Toubkal (Haut Atlas marocain), M. le Professeur F. Vaillant (Grenoble) a récolté des imagos d'Athericidae appartenant à une espece nouvelle. Cette dernière est voisine d'Ibisia marginata (Fabricius, 1781) et d'I. vaillanti Thomas 1982 : elle s'en distingue essentiellement par la coloration de l'aile et la morphologie des genitalia $\sigma$.

\section{Ibisia amicorum n. sp. : description.}

Imago ơ (matériel conservé à sec, piqué).

Téte.

Ensemble brun foncé à poudré gris.

Yeux brun foncé brillant à reflets cuivrés, nettement séparés sur le vertex par un mamelon ocellaire plutôt volumineux, assez large, portant des soies noires et des ocelles brun rouge. Front proéminent, brun noirâtre terne, peu poudré et à forte pilosité noire. Entre les antennes et le clypeus, face à fort

1. Laboratoire d'Hydrobiologie, UA 695 du C.N.R.S., Université Paul Sabatier, 118, route de Narbonne, 31062 Toulouse Cedex, France. poudré gris argenté. Joues pourvues d'une dense pilosité noire. Antennes brun foncé poudré, en particulier le scape et le pédicelle ; arista brun brillant. Clypeus brun brillant à poudré gris, assez saillant mais pas hémisphérique : de profil, son contour est plutôt rectiligne et peut même présenter un début de constriction transversale. Trompe et palpes brun foncé, ces derniers poudrés et à forte pilosité noire. Région postérieure de la tête brun très foncé à poudré gris argenté : moitié supérieure concave à pilosité courte et noire ; moitié inférieure à pruinosité plus marquée et à pilosité plus longue et plus fine, noire à la périphérie - près du bord oculaire, blanche ailleurs.

Thorax.

Entièrement brun foncé, y compris les calus huméraux. Poudré : assez faible dorsalement, plus marqué sur less plẹures. Pilosité : noire dorsalement ; blanche sur les pleures, la région proximale antérieure de la coxa I (et parfois aussi de la coxa II), la totalité de la coxa III et en général une partie des fémurs Il et III ; marron ou noire sur la région distale des coxas I et II et sur le reste des pattes.

Pattes I à III entièrement brun noirâtre, depuis les coxas incluses. Eperons tibiaux : formule de 0-2-2. 


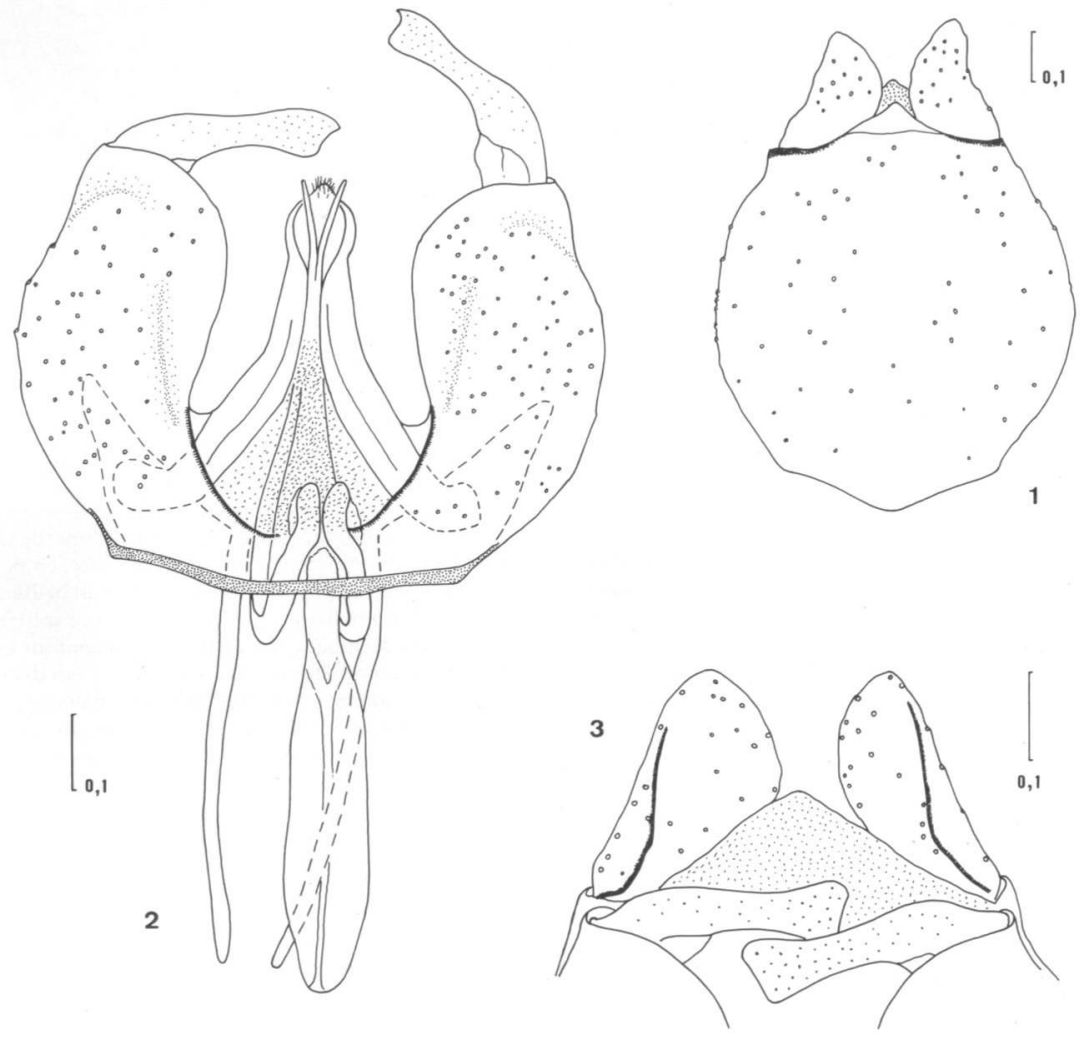

FIG. 1 à 3. Imago o d'Ibisia amicorum n. sp. Echelle en mm.

1 : vue dorsale de l'épandrium et des cerques. 2 et $3:$ genitalia en vue ventrale ( $3:$ dististyles davantage de profil qu'en 2 ). 
Ailes : coloration comportant trois bandes transversales brun noirâtre, pratiquement identiques à celles d'I. marginata et donc un peu moins étendues - et surtout nettement moins intenses - que celles d'I. vaillanti. La bande proximale traverse cependant la cellule basale postérieure. Les zones claires sont relativement peu hyalines, plutôt grisées : l'aile est ainsi moins constrastée que chez $I$, vaillanti.

Balanciers : massue brun foncé, région proximale de la tige jaune brun.

\section{Abdomen.}

Brun foncé. Pilosité : dorsale variant du brun au blanc, ventrale entièrement blanche. Un fort poudré blanc argenté recouvre une fraction de la surface des tergites, fraction décroissante de l'avant vers l'arrière. Le premier tergite, presque entièrement poudré, n'est brun que suivant une bande longitudinale médiane plus étroite que le scutellum. Sur le second tergite, et plus ou moins nettement sur le troisième, un grand triangle isocèle médian brun dont la base correspond à la totalité du bord antérieur du tergite et dont le sommet atteint le bord postérieur de ce dernier - est ménagé entre deux triangles la téraux postérieurs à poudré brillant qui s'étendent jusqu'aux pleures. Sur les tergites suivants, le poudré forme seulement un large liséré postérieur, interrompu ou non au milieu selon les individus. Le poudré est assez uniforme sur la totalité des sternites, mais moins intense que sur les tergites.

\section{Genitalia}

Brun noirâtre, sauf les dististyles, brun jaunâtre ou moyen. Deux caractères sont intermédiaires entre ceux d'I. marginata et d'I. vaillanti (voir Thomas 1974 et 1982) : les cerques, assez variables mais de longueur moyenne ( $f$ ig. 1 et 3 ), et le bord interne du basistyle, modérément proéminent (fig. 2). Le meilleur caractère distinctif, vis-à-vis des genitalia des autres espèces européennes d'Ibisia, est fourni par la morphologie du dististyle, massif et surtout incurvé et très élargi à l'apex, du côté externe ( $f$ ig. 2 et 3). Edéage voisin de ceux d' $I$. marginata et d' $I$. vaillanti.

Taille.

Longueur de l'aile $(\sigma): 6,8$ à $7,4 \mathrm{~mm}$.

Matériel examiné.

4 à sec, piqués, provenant de l'Asif Rerhaïa près du Marabout Sidi Chamarouch (Massif du Toubkal, Haut Atlas, Maroc) à $2310 \mathrm{~m}, 13-$ VII-1954.

\section{Remerclements}

Je suis heureux d'exprimer mes nemerciements amicaux à Monsieur le Professeur F. Vaillant (Grenoble) pour te dor de ces spécimens.

\section{Travaux cités}

Thomas (A.G.B.). 1974. - Diptères torrenticoles peu connus : I. Les Athericidae (larves et imagos) du Sud de la France (Brachycera, Orthorrhapha). Annis Limnol., 10 (1) : 55-84.

Thomas (A.G.B.). 1982. - Diptères torrenticoles peu connus, VIII. Les Athericidae (Ibisia vallanti t. sp.) du Sud de la France (Brachycera, Orthorrhapha). Annls Limnol, 18 (1) : 81-86. 